\title{
Trauma patients with human immunodeficiency virus (HIV): a propensity matched analysis
}

\author{
Jose Covarrubias ${ }^{1}$ - Areg Grigorian ${ }^{2} \cdot$ Catherine M. Kuza ${ }^{2} \cdot$ Matthew Dolich $^{2} \cdot$ Austin Dosch $^{2} \cdot$ Greg G. Kojayan ${ }^{2}$. \\ Patrick Delaplain $^{2} \cdot$ Michael Lekawa $^{2} \cdot$ Jeffry Nahmias ${ }^{2}$ (I)
}

Received: 1 April 2020 / Accepted: 16 May 2020 / Published online: 24 May 2020

○) Springer-Verlag GmbH Germany, part of Springer Nature 2020

\begin{abstract}
Background Given the growing number of people worldwide living with human immunodeficiency virus (HIV), a larger subset of these patients are now susceptible to sustaining a traumatic injury. However, the impact of HIV on outcomes in trauma with modern antiretroviral treatment remains unclear. We hypothesized mortality and rates of infectious and inflammatory complications would be higher in HIV positive (HIV+) trauma patients.

Methods The Trauma Quality Improvement Program was queried to identify trauma patients $\geq 18$ years of age with HIV. Due to the imbalance between HIV+ and HIV negative (HIV-) trauma patients, a 1:2 propensity-matched model was utilized. Matched variables included age, injury severity score, mechanism of injury, systolic blood pressure, pulse rate, Glasgow Coma Scale score, and patient comorbidities.

Results $84 \mathrm{HIV}$ + patients were matched to $168 \mathrm{HIV}$ - patients. Compared to HIV- patients, HIV + patients had no significant differences in mortality rate $(9.5 \%$ vs. $4.8 \%, p=0.144)$ or infectious complications, including pneumonia $(6.0 \%$ vs. $4.2 \%$, $p=0.530)$, urinary tract infection $(1.2 \%$ vs. $1.2 \%, p=1.000)$, or severe sepsis $(1.2 \%$ vs. $0.0 \%, p=0.156)$. However, higher rates of acute respiratory distress syndrome (ARDS) ( $9.5 \%$ vs. $0.6 \%, p<0.001)$ and acute kidney injury (AKI) $(4.8 \%$ vs. $0.0 \%$, $p=0.004)$ were observed.

Conclusion HIV+ trauma patients are not at higher risk of mortality or infectious complications, likely due to the advent and prevalence of combination antiretroviral therapy. However, HIV positivity appears to increase the risk of AKI and ARDS in trauma patients. Further research is needed to confirm this finding to elucidate the etiology underlying this association.
\end{abstract}

Keywords Trauma $\cdot$ HIV $\cdot$ AIDS $\cdot$ Mortality $\cdot$ Complications $\cdot$ Outcomes

\section{Introduction}

As per the Joint United Nations Program on Human Immunodeficiency Virus/Acquired Immunodeficiency Syndrome (HIV/AIDS), there were an estimated 36.9 million people worldwide living with HIV/AIDS in 2017 [1]. Within the United States, the Center for Disease Control and Prevention (CDC) estimates there were approximately 1.1 million

Jeffry Nahmias

jnahmias@hs.uci.edu

1 Department of Surgery, UCSF Fresno, 2823 Fresno St, Fresno, CA, USA

2 Division of Trauma, Burns, Surgical Critical Care and Acute Care Surgery, Department of Surgery, Irvine Medical Center, University of California, 333 The City Blvd West, Suite 1600, Orange, CA 92868-3298, USA people aged $\geq 13$ years living with HIV in 2015 [2]. Interestingly, most people with HIV are between the ages of 45-54, despite a much higher infection rate in younger individuals, indicating that people are surviving much longer with the disease [2]. In fact, since 2010, there has been a $34 \%$ decrease in AIDS-related deaths [3]. With fewer HIV positive $(\mathrm{HIV+})$ patients succumbing to the progression of their disease, there is now a larger subset of these patients at risk for sustaining a traumatic injury. To optimize care for these patients, a keen understanding of the impact HIV has on outcomes in trauma is needed.

To date, the effects of HIV on trauma has been mixed in the literature. Martin et al. showed increased 30-day mortality in trauma patients [4] while other studies demonstrated no association with mortality [6-10]. Similarly, some studies have shown higher renal and pulmonary complications $[5,6]$ as well as higher infectious complications $[5,7,8]$, 
whereas others have demonstrated no difference in renal [7, 8], pulmonary [7, 8], infectious [6], or overall complication rates $[4,9]$ in HIV + trauma patients.

Furthermore, much of the existing literature evaluating the impact of HIV on clinical outcomes in trauma is nearing a decade old [5-8]. Since publication of these studies, the life expectancy in HIV+ patients has improved dramatically with the use of combination antiretroviral therapy (cART) such that the life expectancy gap relative to HIV negative (HIV-) patients is now as low as 8 years [10-12]. In addition, many of the available studies either analyze a small population and/or are conducted at a single center [4-6, 9]. Therefore, we sought to provide a large contemporary descriptive analysis of clinical outcomes in HIV+ trauma patients. We hypothesized that a HIV+trauma cohort would have increased mortality rates, infectious complications (i.e., pneumonia, urinary tract infection (UTI), and sepsis), and inflammatory complications [i.e., acute respiratory distress syndrome (ARDS) and acute kidney injury (AKI)], compared to a propensity-matched HIV- group.

\section{Methods}

This study was approved by the Institutional Review Board at our institution. Furthermore, the study utilized a large national database with de-identified patients, thus no consent was required. The database used was the Trauma Quality Improvement Program (TQIP), a database comprised of a retrospective cohort of trauma patients from participating level I or level II trauma centers in the United States and Canada. TQIP patient inclusion criteria consists of age $\geq 16$, presence of at least one valid trauma International Statistical Classification of Diseases and Related Health Problems (ICD) diagnosis code, blunt or penetrating mechanisms of injury, Abbreviated Injury Scale (AIS) score $\geq 3$, and having data for hospital or emergency department disposition [13]. TQIP patient exclusion criteria consists of patients with advanced directives preventing life-sustaining care or age $\geq 65$ with isolated hip fractures [13]. A retrospective analysis of the TQIP was performed from January 2010 to December 2016 to identify trauma patients who were $\geq 18$ years of age with HIV. Patients considered HIV+ were those with an ICD-9 diagnosis code for HIV: 72 and 79.53. Patients considered HIV - were those without an ICD-9 diagnosis code for HIV.

Due to the observed imbalance between HIV+ and HIV - trauma patients, we elected to perform a propensitymatched analysis using a 1:2 model. This was derived from a logistic regression model in which the dependent variable was HIV. The variables utilized in our model included age, injury severity score (ISS), mechanism of injury, systolic blood pressure (SBP), pulse rate, Glasgow Coma Scale
(GCS) score on admission, and comorbidities such as history of hypertension, chronic obstructive pulmonary disease (COPD), cirrhosis, congestive heart failure (CHF), steroid use, end-stage renal disease (ESRD), and disseminated cancer. Patients with similar propensity scores were matched to compare outcomes among patients with and without HIV. Only cases that were within 0.001 of the estimated logit were selected. This technique of defining the closeness of a matched case is termed caliper matching and is a validated method of emulating randomization in observational studies [14]. Once propensity scores were calculated for each case, one HIV+ and two HIV - matched trauma patients were included for further analysis. If a close match was not available for a HIV+ trauma patient, they were excluded from analysis.

We performed bivariate analyses for all variables to confirm a successfully matched cohort. This was done with either a chi-square or Mann-Whitney- $U$ test for categorial and continuous variables, respectively. The primary outcome was in-hospital mortality. Secondary outcomes included infectious and inflammatory complications such as AKI, ARDS, pneumonia, and UTI as well as other in-hospital complications. Other measured outcomes included total hospital length of stay (LOS), ventilator days and intensive care unit (ICU) LOS. All $p$-values were two-sided, with a statistical significance level of $<0.05$. All analyses were performed with IBM SPSS Statistics for Windows (Version 24, IBM Corp., Armonk, NY).

\section{Results}

\section{Demographics of HIV+ trauma patients}

From 1,403,466 trauma patients, 94 (0.007\%) patients were HIV+ . After propensity-score matching, $84 \mathrm{HIV}+$ trauma patients were compared to a cohort of $168 \mathrm{HIV}$ - trauma patients. There were 10 patients that did not fit into our propensity-matched model and were thus excluded from analysis (Fig. 1). Compared to a matched cohort of HIV - patients, the HIV + patients had no differences in demographics, injury patterns, or vital signs upon hospital arrival. This confirmed successful matching between the two groups. Hypertension was the most common comorbid condition in both groups, followed by COPD, and diabetes. Traumatic brain injury (TBI) was the most common injury in both groups followed by injuries to the lower extremity (Table 1).

\section{Clinical outcomes in HIV+ trauma patients}

There was no difference observed in mortality rate $(4.8 \%$ vs $9.5 \%, p=0.144)$ or in the rate of infectious complications 
Fig. 1 Selection of patients for the propensity matched analysis. *Patients that did not fit into the propensity-matched model and were excluded from analysis

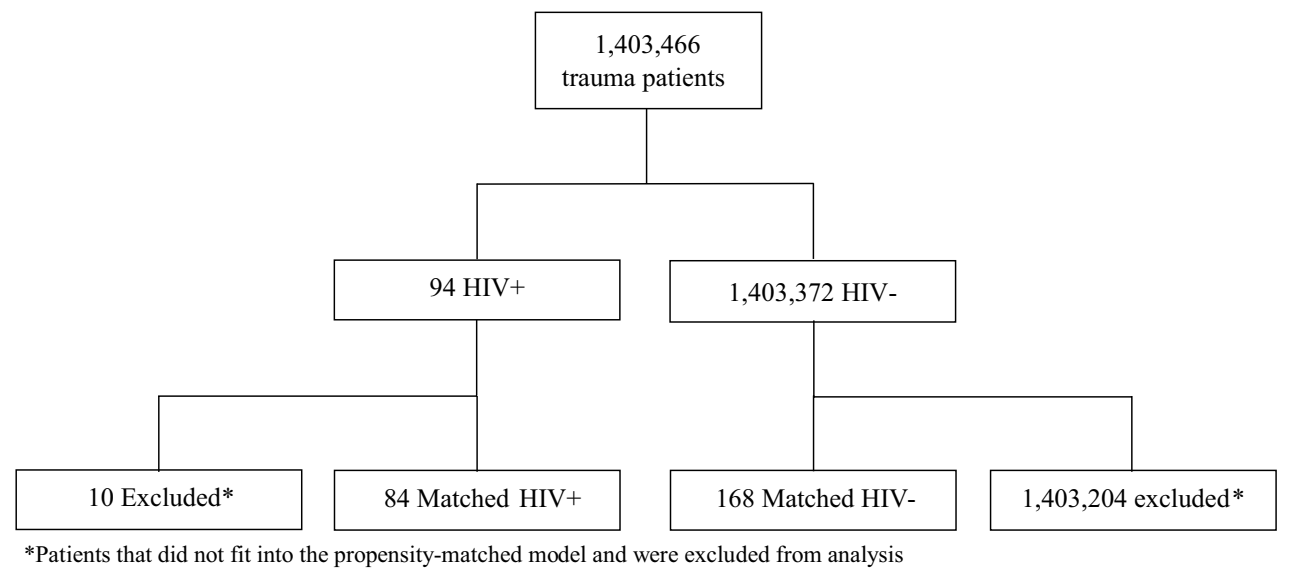

including UTI ( $1.2 \%$ vs $1.2 \%, p=1.000)$, pneumonia $(6.0 \%$ vs $4.2 \%, p=0.530)$, and sepsis $(1.2 \%$ vs $0.0 \%, p=0.156)$ between the HIV+ and HIV - group. However, higher rates of ARDS (0.6\% vs. $9.5 \%, p<0.001)$ and AKI $(0.0 \%$ vs. $4.8 \%, p=0.004)$ were observed in the HIV+ group relative to the HIV - group. The LOS, ICU LOS, and median ventilator days did not differ between the two groups (Table 2).

\section{Discussion}

Though once considered a fatal disease, HIV/AIDS now more closely resembles a chronic disease with the advent and rise of cART medications [3, 15, 16]. In this study, we evaluated the clinical outcomes in this modern-day $\mathrm{HIV}+$ trauma patient population and compared our findings to those reported in earlier studies. Using seven years of data derived from the TQIP database, we demonstrated no differences in mortality or rates of infectious complications between HIV + and HIV - trauma patients. However, the HIV+ group was found to have higher rates of AKI and ARDS.

Prior literature generally has shown no association between HIV and mortality in trauma patients [5-8]. However, a recent study by Martin et al. evaluating trauma patients in Rwanda found HIV to be associated with increased 30-day mortality although no in-hospital mortality was reported [4]. Our study supports the findings of earlier domestic studies but appears to contrast the findings of the international study by Martin et al. It should be noted that direct comparisons between this study and the one by Martin et al. are difficult given the geographic disparities as well as the differing primary outcome of interest. However, their finding of no in-hospital mortality appears to support our results.

The association between HIV and development of infectious complications in trauma patients has been mixed, with some studies demonstrating higher $[5,7,8]$ rates and others demonstrating no difference [6] in the rate of infectious complications like pneumonia, UTI, bacteremia, and sepsis. However, these studies are relatively old with data ranging from 1989 to 2006, a time when treatment for HIV was not widely available. In fact, the CDC estimates only $28 \%$ of all HIV+ patients in the U.S. had achieved viral suppression by 2010 [17]. The only research addressing infectious complications in modern-day HIV+ trauma patients has been through relatively small single center or multi-center studies conducted outside of the U.S. Both, Martin et al., who evaluated adult $\mathrm{HIV}+$ trauma patients in Rwanda, and McPherson et al., who evaluated adult HIV+ patients with penetrating abdominal trauma in South Africa, demonstrated no difference in infectious complications [4, 9]. Similarly, we did not find a difference in infectious complications when comparing U.S. HIV + to HIV - trauma patients. This is likely reflective of the growing prevalence of cART and should thus be considered as physicians counsel patients and/or make decisions regarding management of $\mathrm{HIV}+$ trauma patients.

The association between HIV and ARDS/AKI in trauma has varied in previous reports [5-8]. Here we reported a higher rate of ARDS and AKI in HIV+ trauma patients, a finding that supports the work by Stawicki et al. and Duane et al. [5,6]. The mechanism for this might be related to the chronic inflammatory state that remains in HIV+ patients despite adequate viral suppression from cART [18]. As a known inducer of systemic inflammation [19], traumatic injury in $\mathrm{HIV}+$ patients may serve to further augment the inflammatory state already present. This can amplify the activity of neutrophils known to be a component of the chronic inflammatory state observed in HIV [20]. Neutrophil activation is known to be key in the pathogenesis of ARDS [21] so additional neutrophil involvement may make HIV + trauma patients susceptible for the development of ARDS. This augmented inflammatory state may also amplify pre-existing renal tissue injury and contribute to the development of AKI. However, the mechanism for higher rates of 
Table 1 Demographics of HIV+ trauma patients

\begin{tabular}{|c|c|c|c|}
\hline Characteristic & Non-HIV $(n=168)$ & HIV $(n=84)$ & $p$ value \\
\hline $\begin{array}{l}\text { Age, year, median } \\
\text { (IQR) }\end{array}$ & $50.0(42)$ & $50.0(13)$ & 0.824 \\
\hline Male, $n(\%)$ & $131(78.0 \%)$ & $67(79.8 \%)$ & 0.745 \\
\hline ISS, median (IQR) & $13.5(13)$ & $10.0(13)$ & 0.824 \\
\hline Blunt trauma, $n(\%)$ & $152(90.5 \%)$ & $76(90.5 \%)$ & 1.000 \\
\hline $\begin{array}{l}\text { Penetrating trauma, } n \\
(\%)\end{array}$ & $16(9.5 \%)$ & $8(9.5 \%)$ & 1.000 \\
\hline \multicolumn{4}{|l|}{ Vitals/GCS } \\
\hline $\begin{array}{l}\text { Lowest SBP within } \\
24 \mathrm{~h} \text {, median (IQR) }\end{array}$ & $138(34)$ & $132(36)$ & 0.755 \\
\hline $\begin{array}{l}\text { Pulse rate, median } \\
\text { (IQR) }\end{array}$ & $88.5(30)$ & $88.0(20)$ & 0.964 \\
\hline GCS, median (IQR) & $15(0)$ & $15(1)$ & 1.000 \\
\hline \multicolumn{4}{|l|}{ Comorbidities, $n(\%)$} \\
\hline Diabetes & $27(16.1 \%)$ & $12(14.3 \%)$ & 0.712 \\
\hline Hypertension & $50(29.8 \%)$ & $25(29.8 \%)$ & 1.000 \\
\hline COPD & $31(18.5 \%)$ & $15(17.9 \%)$ & 0.908 \\
\hline $\begin{array}{l}\text { Congestive heart } \\
\text { failure }\end{array}$ & $6(3.6 \%)$ & $3(3.6 \%)$ & 1.000 \\
\hline Cirrhosis & $5(3.0 \%)$ & $3(3.6 \%)$ & 0.799 \\
\hline Steroid use & $0(0.0 \%)$ & $0(0.0 \%)$ & 1.000 \\
\hline Chronic renal failure & $8(4.8 \%)$ & $3(3.6 \%)$ & 0.663 \\
\hline Disseminated cancer & $2(1.2 \%)$ & $1(1.2 \%)$ & 1.000 \\
\hline \multicolumn{4}{|l|}{ Injuries, $n(\%)$} \\
\hline Traumatic brain injury & $78(46.4 \%)$ & $36(42.9 \%)$ & 0.591 \\
\hline Spine & $42(25.0 \%)$ & $16(19.0 \%)$ & 0.290 \\
\hline Upper extremity & $37(22.0 \%)$ & $14(16.7 \%)$ & 0.318 \\
\hline Lower extremity & $56(33.3 \%)$ & $32(38.1 \%)$ & 0.455 \\
\hline Lung & $38(22.6 \%)$ & $18(21.4 \%)$ & 0.830 \\
\hline Heart & $0(0.0 \%)$ & $0(0.0 \%)$ & 1.000 \\
\hline Esophagus & $1(0.6 \%)$ & $0(0 \%)$ & 0.479 \\
\hline Stomach & $2(1.2 \%)$ & $1(1.2 \%)$ & 1.000 \\
\hline Small intestine & $6(3.6 \%)$ & $2(2.4 \%)$ & 0.611 \\
\hline Colorectal & $4(2.4 \%)$ & $0(0.0 \%)$ & 0.154 \\
\hline Pancreas & $2(1.2 \%)$ & $0(0.0 \%)$ & 0.315 \\
\hline Liver & $9(5.4 \%)$ & $6(7.1 \%)$ & 0.572 \\
\hline Spleen & $11(6.5 \%)$ & $7(8.3 \%)$ & 0.604 \\
\hline Kidney & $2(1.2 \%)$ & $1(1.2 \%)$ & 1.000 \\
\hline
\end{tabular}

$H I V$ human immunodeficiency virus, $I Q R$ interquartile range, $I S S$ injury severity score, $S B P$ systolic blood pressure, GCS Glasgow coma scale, $C O P D$ chronic obstructive pulmonary disease

$\mathrm{AKI}$ is likely multifactorial since $\mathrm{AKI}$ in $\mathrm{HIV}+$ patients has typically been shown to have two or more contributing etiological factors such as infection, drug nephrotoxicity, and/ or decreased renal perfusion [22]. The nephrotoxic effects of antiretroviral agents such as atazanavir, lopinavir, indinavir, and tenofovir are well documented [23-25] and may occur insidiously [26]. It is possible that traumatic injury in HIV + patients led to decreased renal perfusion from hypovolemia, exacerbating pre-existing renal injury and increasing the likelihood for AKI. More research is needed to evaluate the pathophysiology behind the increased rates of ARDS and AKI among HIV+trauma patients. Clinicians should be aware of this finding to attempt to mitigate this through vigilant monitoring and/or targeted interventions.

As a large retrospective database study, there are inherent limitations to this work such as reporting bias and coding errors. The estimated prevalence of HIV in the US population in 2015 was $0.3 \%$, which is much higher than the observed prevalence in this study. This could indicate a significant population of missed HIV+ patients in the cohort. Another limitation is in how HIV positivity was determined. To be considered HIV + for this study, an ICD-9 diagnosis code for HIV must have been present. However, HIV is not a mandated comorbid condition. Therefore, it is possible HIV+ patients went unaccounted for, potentially further explaining the much lower prevalence of HIV in our study relative to the United States general population. Additionally, the TQIP database does not provide information on a patient's CD4 count, viral load, or treatment, which creates a non-homogenous population of HIV+ patients. Furthermore, we do not have any information regarding baseline renal function or prior history of infectious complications in our patient population, which may be serving as potential confounders. An additional important point to consider is the finding of no statistically significant difference in mortality rate between the two study groups despite the rate being nearly double in the HIV+ group. This may represent a type II error and should be investigated further with a larger multicenter study. Finally, as a retrospective study, our findings cannot be interpreted as causation. Our findings can only serve as markers of a correlation that merits future exploration.

\section{Conclusion}

HIV + trauma patients had a similar rate of mortality and infectious complications compared to a matched population of HIV - trauma patients. This may be due to the advent of cART. However, HIV positivity is associated with an increased incidence of AKI and ARDS in trauma patients. Future clinical studies and basic science research investigating biochemical and/or physiological pathways that may predispose HIV+ patients to these inflammatory complications are warranted. 
Table 2 Clinical outcomes in HIV+ trauma patients

\begin{tabular}{|c|c|c|c|}
\hline Outcome & Non-HIV $(n=168)$ & HIV $(n=84)$ & $p$ value \\
\hline LOS, days, median (IQR) & $5.0(7)$ & $6.0(9)$ & 0.752 \\
\hline ICU, days, median (IQR) & $3.0(3)$ & $3(6)$ & 0.952 \\
\hline Ventilator, days, median (IQR) & $2.0(6)$ & $3.0(5)$ & 0.674 \\
\hline \multicolumn{4}{|l|}{ Complications, $n(\%)$} \\
\hline Myocardial infarction & $0(0.0 \%)$ & $0(0.0 \%)$ & 1.000 \\
\hline Cardiac arrest & $2(1.2 \%)$ & $1(1.2 \%)$ & 1.000 \\
\hline Cerebrovascular accident & $2(1.2 \%)$ & $0(0.0 \%)$ & 0.315 \\
\hline Pneumonia & $7(4.2 \%)$ & $5(6.0 \%)$ & 0.530 \\
\hline Ventilator associated pneumonia & $0(0.0 \%)$ & $0(0.0 \%)$ & 1.000 \\
\hline Unplanned intubation & $2(1.2 \%)$ & $1(1.2 \%)$ & 1.000 \\
\hline Acute respiratory distress syndrome & $1(0.6 \%)$ & $8(9.5 \%)$ & $<0.001$ \\
\hline Acute kidney injury & $0(0.0 \%)$ & $4(4.8 \%)$ & 0.004 \\
\hline Deep venous thrombosis & $4(2.4 \%)$ & $0(0.0 \%)$ & 0.154 \\
\hline Pulmonary embolism & $1(0.6 \%)$ & $1(1.2 \%)$ & 0.616 \\
\hline Decubitus ulcer & $2(1.2 \%)$ & $3(3.6 \%)$ & 0.201 \\
\hline Drug/alcohol withdrawal & $5(3.0 \%)$ & $4(4.8 \%)$ & 0.471 \\
\hline Compartment syndrome, extremity & $0(0.0 \%)$ & $1(1.2 \%)$ & 0.156 \\
\hline Graft/flap failure & $0(0.0 \%)$ & $0(0.0 \%)$ & 1.000 \\
\hline Unplanned return to OR & $1(0.6 \%)$ & $1(1.2 \%)$ & 0.616 \\
\hline Unplanned ICU & $4(2.4 \%)$ & $1(1.2 \%)$ & 0.523 \\
\hline Superficial infection & $0(0.0 \%)$ & $0(0.0 \%)$ & 1.000 \\
\hline Deep site infection & $1(0.6 \%)$ & $0(0.0 \%)$ & 0.479 \\
\hline Urinary tract infection & $2(1.2 \%)$ & $1(1.2 \%)$ & 1.000 \\
\hline Organ space infection & $0(0.0 \%)$ & $(0.0 \%)$ & 1.000 \\
\hline Osteomyelitis & $0(0.0 \%)$ & $0(0.0 \%)$ & 1.000 \\
\hline CAUTI & $0(0.0 \%)$ & $0(0.0 \%)$ & 1.000 \\
\hline CRBSI & $0(0.0 \%)$ & $1(1.2 \%)$ & 0.156 \\
\hline CLABSI & $0(0.0 \%)$ & $0(0.0 \%)$ & 1.000 \\
\hline Severe sepsis & $0(0.0 \%)$ & $1(1.2 \%)$ & 0.156 \\
\hline Other & $38(22.6 \%)$ & $12(14.3 \%)$ & 0.118 \\
\hline Mortality, $n(\%)$ & $8(4.8 \%)$ & $8(9.5 \%)$ & 0.144 \\
\hline
\end{tabular}

$H I V$ human immunodeficiency virus, $L O S$ length of stay, $I Q R$ interquartile range, $I C U$ intensive care unit, $O R$ operating room, $C A U T I$ catheter-associated urinary tract infection, CRBSI catheter-related bloodstream infection, $C L A B S I$ central line-associated bloodstream infection
Author contributions JC, JN, AG were involved in the development of the study design as well as completion of the manuscript. AG compiled the necessary study statistics. All authors were involved in the analysis of data and critical revisions to the manuscript.

Funding This research did not receive any specific grant from funding agencies in the public, commercial, or not-for-profit sectors.

\section{Compliance with ethical standards}

Conflict of interest The authors report no conflict of interest, financial or otherwise.

Informed consent This retrospective study involved humans but since a national database was used with de-identified patients, risk was minimal to participants. No consent was required. 


\section{References}

1. UNAIDS Data 2018. Joint United Nations Programme on HIV/ AIDS (UNAIDS). https://www.unaids.org/sites/default/files/ media_asset/unaids-data-2018_en.pdf. Published July 2018. Accessed 22 Jan 2019.

2. CDC. Estimated HIV incidence and prevalence in the United States, 2010-2015. HIV Surveillance Supplemental Report. 2018;23(1). https://www.cdc.gov/hiv/library/reports/hiv-surve illance.html. Published March 2018. Accessed 22 Jan 2019.

3. WHO HIV Update 2017. World Health Organization (WHO). https://www.who.int/hiv/data/2017_global_summary_web_v11. pptx. Accessed 3 Jun 2019.

4. Martin AN, Byiringiro JC, Petroze RT, Nkeshimana M, Byiringiro F, Calland JF. Assessing the impact of HIV status on injury outcomes: a multicenter study of trauma patients in Rwanda. Surgery. 2019;165(2):444-9.

5. Stawicki SP, Hoff WS, Hoey BA, Grossman MD, Scoll B, Reed JF 3rd. Human immunodeficiency virus infection in trauma patients: where do we stand? J Trauma. 2005;58(1):88-93.

6. Duane TM, Sekel S, Wolfe LG, Malhotra AK, Aboutanos MB, Ivatury RR. Does HIV influence outcomes after trauma? J Trauma. 2008;65(1):63-5.

7. Morrison CA, Wyatt MM, Carrick MM. Effects of human immunodeficiency virus status on trauma outcome: a review of the national trauma database. Surg Infect (Larchmt). 2010;11(1):41-7.

8. Patel MS, Malinoski DJ, Nguyen XM, Hoyt DB. The impact of select chronic diseases on outcomes after trauma: a study from the National Trauma Data Bank. J Am Coll Surg. 2011;212(1):96-104.

9. McPherson D, Neuhaus V, Dhar R, Edu S, Nicol AJ, Navasaria $\mathrm{PH}$. The effect of human-immunodeficiency virus status on outcomes in penetrating abdominal trauma: an interim analysis. World J Surg. 2018;42:2412-20.

10. Marcus JL, Chao CR, Leyden WA, et al. Narrowing the gap in life expectancy between HIV-infected and HIV-uninfected individuals with access to care. J Acquir Immune Defic Syndr. 2016;73(1):39-46.

11. Wandeler G, Johnson LF, Egger M. Trends in life expectancy of HIV-positive adults on antiretroviral therapy across the globe: comparisons with general population. Curr Opin HIV AIDS. 2016;11(5):492-500.

12. Teeraananchai S, Kerr SJ, Amin J, et al. Life expectancy of HIVpositive people after starting combination antiretroviral therapy: a meta-analysis. HIV Med. 2017;18(4):256-66.
13. Newgard CD, Fileds JJ, Wu L, et al. Methodology and analytic rationale for the American College of Surgeons Trauma Quality Improvement Program. J Am Coll Surg. 2013;216(1):147-57.

14. Austin PC. Optimal caliper widths for propensity-score matching when estimating differences in means and differences in proportions in observational studies. Pharm Stat. 2011;10(2):150-61.

15. Barré-Sinoussi F, Ross AL, Delfraissy JF. Past, present, and future: 30 years of HIV research. Nat Rev Microbiol. 2013;11:877-83.

16. CDC. Monitoring selected national HIV prevention and care objectives by using HIV surveillance data-United States and 6 dependent areas, 2016. HIV Surveillance Supplemental Report 2018;23(No. 4). https://www.cdc.gov/hiv/library/reports/hiv-surve illance.html. Published June 2018. Accessed 3 Jul 2019.

17. CDC. Vital signs: HIV prevention through care and treatmentUnited States. MMWR. 2011;60(47):1618-23.

18. Rosenberg AZ, Naicker S, Winkler CA, Kopp JB. HIV-associated nephropathies: epidemiology, pathology, mechanisms and treatment. Nat. Rev. Nephrol. 2015;11:150-160.

19. Lenz A, Franklin GA, Cheadle WG. Systemic inflammation after trauma. Injury. 2007;38 (12):1336-45.

20. Yaseen MM, Abuharfeil NM, Yaseen MM, Shabsoug BM. The role of polymorphonuclear neutrophils during HIV-1 infection. Arch Virol. 2018;163(1):1-21.

21. Pierrakos C, Karanikolas M, Scolletta S, Karamouzos V, Velissaris D. Acute respiratory distress syndrome: pathophysiology and therapeutic options. J Clin Med Res. 2012;4(1):7-16.

22. Roe J, Campbell LJ, Ibrahim F, et al. HIV care and the incidence of acute renal failure. Clin Infect Dis. 2008;47:242-249.

23. Yombi JC, Pozniak A, Boffito M, Jones R, Khoo S, Levy J, et al. Antiretrovirals and the kidney in current clinical practice: renal pharmacokinetics, alterations of renal function and renal toxicity. AIDS 2014; 28:621-632.

24. Mocroft A, Kirk O, Reiss P, De Wit S, Sedlacek D, Beniowski M, et al. Estimated glomerular filtration rate, chronic kidney disease and antiretroviral drug use in HIV-positive patients. AIDS 2010; 24:1667-1678.

25. Ryom L, Mocroft A, Kirk O, Worm SW, Kamara DA, Reiss $\mathrm{P}$, et al. Association between antiretroviral exposure and renal impairment among HIV-positive persons with normal baseline renal function: the D:A:D study. J Infect Dis 2013; 207:1359- 1369.

26. Campos P, Ortiz A, Soto K. HIV and kidney diseases: 35 years of history and consequences. Clin Kidney J. 2016;9(6):772-781. 